\title{
45. COMMISSION DES CLASSIFICATIONS SPECTRALES ET INDICES DE COULEUR A PLUSIEURS BANDES
}

President: W.P. Bidelman.

\author{
Reports of Meetings
}

SECRETARY: V.C. Reddish.

\section{Business Meeting, 23 August 1967}

DRAFT REPORT

The Draft Report was approved as submitted, subject to the addition of several supplementary reports which appear as an Appendix to the present document.

\section{OFFICERS}

The President reported that the following recommendations were being made for the Commission's officers for the next triennium:

President: Ch. Fehrenbach.

Vice-President: B. E. Westerlund.

Organizing Committee: W.P. Bidelman, E.K. Haradze, C.O. R. Jaschek, H.L. Johnson, W.W. Morgan.

\section{CATALOGUES}

M.F. McCarthy reported that the Vatican Observatory was setting up a card index of references to emission-line stars contained in various published catalogues. C.O. R. Jaschek and K. G. Henize reported similar reference catalogues in preparation. A working group consisting of these three investigators was appointed to coordinate these efforts and to arrange for eventual publication.

W.P. Bidelman expressed the hope that positions for all new discoveries would be given using the equinox of 1900 . M.F. McCarthy stated that at least some coordinates should always be given, even if only X, Y positions on the Palomar Sky Survey charts. W.P. Bidelman spoke of the need for a general reference catalogue giving full bibliographic data for individual stars. About $10^{6}$ stars would be involved, and the resources of a large institution such as NASA would certainly be required to provide such a centralized information service. Several members of the Commission expressed interest in, and support for, this proposal.

\section{SPECTRAL ATLASES}

W.W. Morgan reported that the new Yerkes spectral atlas will be ready about July 1968, and also that the Arizona-KPNO spectral atlas, in which he is also somewhat involved, should be ready at approximately the same time. Ch. Fehrenbach's objective-prism spectral atlas, with a dispersion of $80 \AA / \mathrm{mm}$, is nearly completed. Work is also continuing on the Córdoba spectral atlas at a dispersion of $42 \AA / \mathrm{mm}$. It was stated by W.C. Seitter that her Bonn objective-prism spectral atlas was very near completion, and that it would soon be available. The dispersions involved are $240 \AA / \mathrm{mm}$, $645 \AA / \mathrm{mm}$, and $1280 \AA / \mathrm{mm}$. The President drew attention to the high quality of the spectra reproduced in this work.

\section{STANDARDS}

The need for further faint spectral standards was discussed at some length, and it was pointed out that the extensive work on faint selected-area stars already carried out by N.G. Roman could well 
provide the basis for the selection of a substantial number of standard regions. This problem was referred to the officers of the Commission for further action.

\section{COLLOQUIA AND SYMPOSIA}

It was suggested that the Commission consider the advisability of sponsoring meetings on the subjects of 'Stellar Rotation' and 'Automated Spectral Classification' within the next few years.

\section{Scientific Meetings, 24, 25, 29 August 1967}

The Commission participated in three scientific sessions, the first of which was a joint meeting with Commission 28. In view of the fact that the papers given at these sessions will shortly be published, and also since it is very difficult to adequately summarize the lively discussions that took place at these sessions, it is thought best to simply acknowledge here the presentation of the following papers:

W.W. Morgan: 'Stellar compositions of giant galaxies'.

H. Spinrad: 'Spectrophotometry and stellar content of the nuclei of galaxies'.

J.G. Davies: 'Stellar diameters determined by use of the Brown-Twiss interferometer'.

C.J. Durrant: 'Cambridge narrow-band studies of silicon stars'.

A.G.D. Philip: 'Photoelectric photometry of field horizontal-branch stars'.

J. A. Graham: 'Photoelectric photometry of globular cluster horizontal-branch stars'.

D. L. Crawford: 'Narrow-band photometry of peculiar B stars'.

H.J. Wood: 'Photoelectric studies of spectrum variability in peculiar A stars'.

W.P. Bidelman: 'The detection and photometry of weak-metal stars'.

E.K. Haradze: 'Spectral classification work at the Abastumani Observatory'.

P. Pesch: 'Studies of M dwarfs in the Hyades'.

P.J. Treanor: 'Studies of M dwarfs in the Pleiades'.

W.P. Bidelman: 'The work of the Michigan Curtis Schmidt telescope at Cerro Tololo, Chile'.

$K$. Gyldenkerne: 'Narrow-band photometry of late-type stars'.

J. Rousseau: 'Six-color observations of late-type main sequence stars'.

P.B. Boyce: 'Spectral scans and photometry of late-type dwarfs'.

\section{Appendix}

\section{MATERIAL SUPPLEMENTARY TO DRAFT REPORT}

A. Beer (Cambridge): Work has been carried out at Cambridge to assemble the material for a reduction to a unified system of all measurements of indicators of equivalent width of Balmer lines in several thousand early-type stars, essentially O7-B9-dealt with in about sixty observational series-that have so far been listed in the existing literature, covering some three decades, or have been supplied by colleagues from their unpublished observations. The dependence of these Balmerline intensities on the different instruments and observational methods, as well as on apparent brightness, spectrum, luminosity class, the system of assigned weights, on the errors due to photographic, spectroscopic and random effects, etc.-altogether nine independent parameters-is being analysed in joint work with Professor Th. Schmidt-Kaler in Bonn. A complete computer analysis of the intercorrelation between these series, and their necessary adjustment, provides corrected standard values for all of these stars. The corresponding distance scale is calibrated with the aid of clusters. It is hoped that the final outcome of this homogenization will be improved insight into the structural features of the Milky Way in our neighborhood, spiral arms or otherwise, out to about 4 $\mathrm{kpc}$ and beyond.

J.S. Neff (State U. of Iowa): An intermediate-bandpass photometric system of broad scope has 
been established under the direction of $\mathrm{Dr}$ W.W. Morgan; the fundamental work was done at the McDonald Observatory in the years 1963-64. Laboratory calibrations were carried out using the facilities of the Washburn Observatory and the spectroscopy department of the University of Wisconsin. Details of this work are described by Neff and Travis (Astr. J. 72, 48, 1967), who also describe a new reduction method that yields a substantial increase in accuracy. Some applications of this system, which is called the CHI system, have been made by Morgan and Neff (Astr. J. 69, 145, 1964) and Neff (Astr. J. 71, 202, 1966). More detailed descriptions of these applications are in preparation. At the University of Iowa Observatory the CHI system is used with a 24-inch Cassegrain reflector and an integrating digital photometer. Tests have indicated that observations can be made of stars as faint as $m_{0.55}=14$.

A method has been developed to derive $T_{e}, g$, blocking by lines in the various bandpasses, and reddening for RR Lyrae stars under the assumption that the variable is in a quasi-stationary state near minimum light. This method can also be applied to non-variable stars of spectral types earlier than G8. For stars of later type empirical methods must be used.

V. Straižis (Vilnius Observatory): For the past several years I have been attempting to establish a new multicolor system that will permit a purely photometric two-dimensional classification of stars and a determination of interstellar absorption. Preliminary results have been given in Trans. $I A U .12 \mathrm{~B}, 261,1966$. The first part of the work consisted of a theoretical analysis of stellar energy curves of stars of different spectral types and luminosity classes, as determined by various investigators. The properties of different magnitude systems with intermediate bandwidths were examined in the wavelength range $\lambda \lambda 3000-6500$. As a result a seven-color system with central wavelengths $\lambda 3500(\mathrm{U}), \lambda 3750(\mathrm{P}), \lambda 4050(\mathrm{X}), \lambda 4600(\mathrm{Y}), \lambda 5100(\mathrm{Z}), \lambda 6200(\mathrm{~T})$, and $\lambda 6400(\mathrm{~S})$ and with halfwidths of the order of $200 \AA$ was chosen. The system seems to render possible the purely photometric determination of spectral and luminosity classes for B to $M$ stars, the estimation of their metallicity, and the unambiguous evaluation of interstellar absorption for each individual star. Only five magnitudes, U, P, X, Y, Z, are needed to classify the $\mathrm{O}, \mathrm{B}, \mathrm{A}$, and F stars, while five others, $\mathrm{X}, \mathrm{Y}$, $Z, T, S$, are adequate to classify the $K$ and $M$ stars. For the classification of the G-type stars a combination of both five-color systems may be necessary. The classification is accomplished by different versions of the Q method as used by the Walravens, Borgman, Strömgren and Golay. The system can be used with either interference or glass filters, which makes it simple to adapt it to photographic methods. Observational tests of the new system are being carried out both in Vilnius and in the Crimea. An indirect confirmation of the system's efficiency has been obtained by comparison with other multicolor systems of intermediate bandwidth.

The system is expected to be useful in enabling two- or three-dimensional classification of faint stars in densely crowded fields of the Milky Way where it is difficult to obtain objective-prism spectra. 